\title{
Destructive Tomography of Red Cabbage and Swiss Cheese: FIB-SEM Themed Educational Outreach
}

\author{
Konrad Rykaczewski, ${ }^{1 *}$ Abigail A. Howell, ${ }^{2}$ and Maria Wieczynska ${ }^{3}$ \\ ${ }^{1}$ School for Engineering of Matter, Transport and Energy, Arizona State University, \\ Tempe, 85287 \\ ${ }^{2}$ LeRoy Eyring Center for Solid State Science, Arizona State University, Tempe, 85287 \\ ${ }^{3}$ W.P. Carey School of Business, Arizona State University, Tempe, 85287
}

*konradr@asu.edu

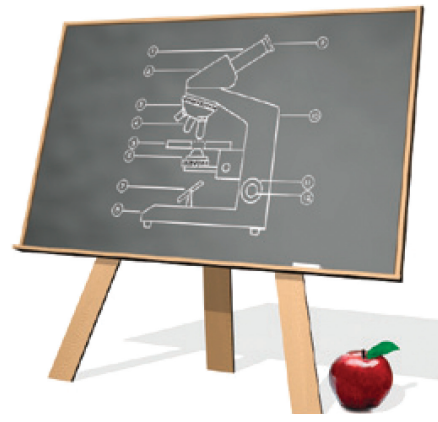

\begin{abstract}
In this article we describe a simple, scalable, low-cost, and hands-on activity that introduces students to microscopy and "destructive tomography." Specifically, we show that 2D images of sequential cross sections of several foods can be quickly reconstructed into a volumetric data set, shared, and interactively explored online using simple and fast image processing. We describe the entire process and present the results of destructive tomography of red cabbage and Swiss cheese as well as describe our experience with implementation of this activity in a local middle school.
\end{abstract}

\section{Introduction}

One of the traditional ways to excite young students about science and technology is to use a microscope to reveal to them features that are, for example, too small to see with a naked eye or hidden within a material. A common example of such outreach activity can include showing a group of students what an insect looks like up close when imaged with a scanning electron microscope (SEM). However, limited access to these tools and safety concerns often restrict such outreach activities to a short demonstration by an experienced operator. In the last decade numerous routes for resolving these issues and expanding the role of microscopy in $\mathrm{K}-12$ education have been proposed. Some high schools have received donations and acquired microscopes for their own campuses [1-5]. Alternatively, $\mathrm{K}-12$ schools located in vicinity of higher education institutions can collaborate with universities to access their microscopy facilities [1, 6-7]. Budget-constrained schools have taken advantage of technological advances such as remote online access and mobile table-top SEMs. For example, projects like Bugscope [8], Project ExCEL [9], and nanoManipulator [10] allow K-12 students to remotely access SEM and scanning probe microscopes. Other efforts bring portable light optical microscopes [11-12] and table-top SEMs [6] directly to the students. However, hands-on activities related to imaging, especially in 3D, which could be performed by a large number of students simultaneously, are rare.

In the last decade, the slice-and-view tomographic method employing a focused ion beam (FIB) instrument combined with an scanning electron microscope (SEM) (a FIB-SEM) [13-14] became a common way to characterize the 3D geometry and composition of a variety of materials from the nanoscale $(\mathrm{nm})$ to the microscale $(\mu \mathrm{m})$ [15-18]. In this article we describe a simple, scalable, low-cost, hands-on activity that introduces students to microscopy and tomography. Specifically, we describe how students can perform macroscale "destructive tomography" of different morphologically rich food items.

The schematic in Figure 1 illustrates the experimental procedure consisting of sequential "slicing" away of a thin section

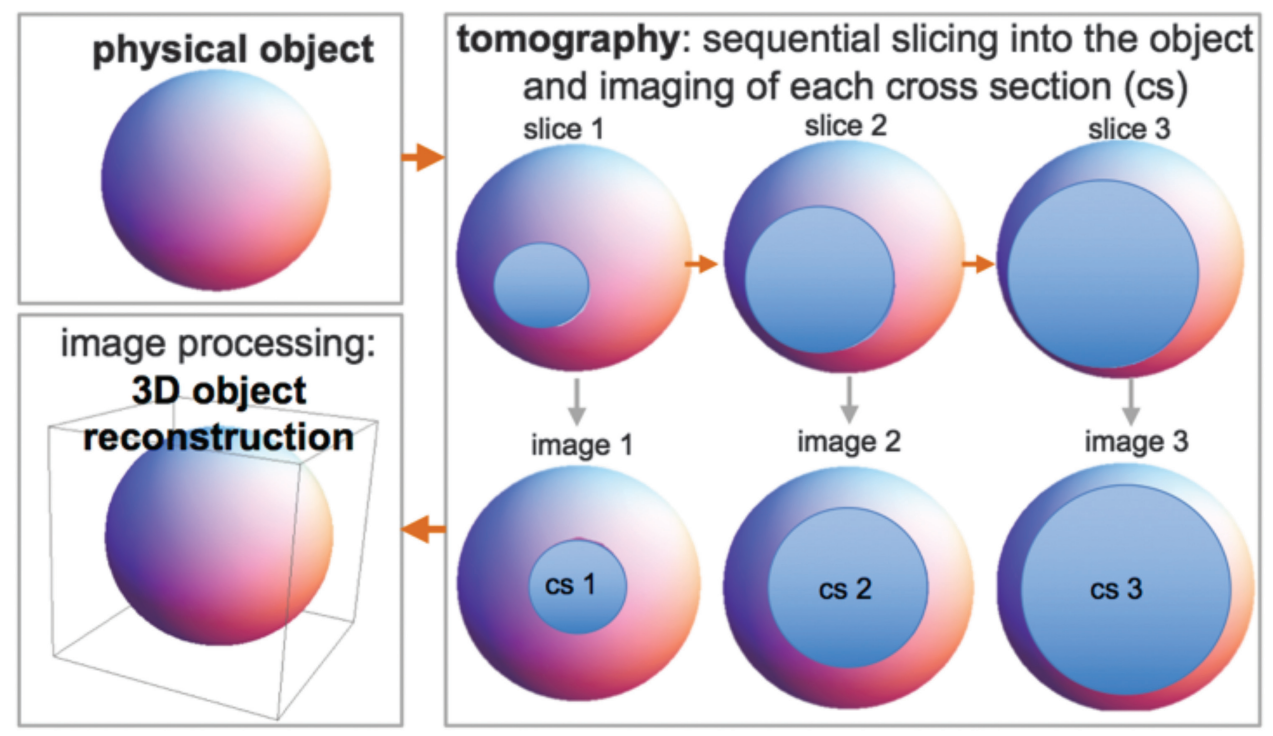

Figure 1: Schematic of a typical "destructive tomography" procedure. After each slice an image is acquired and stored. Reconstruction software assembles the images into a 3D space that may be explored in various ways. 

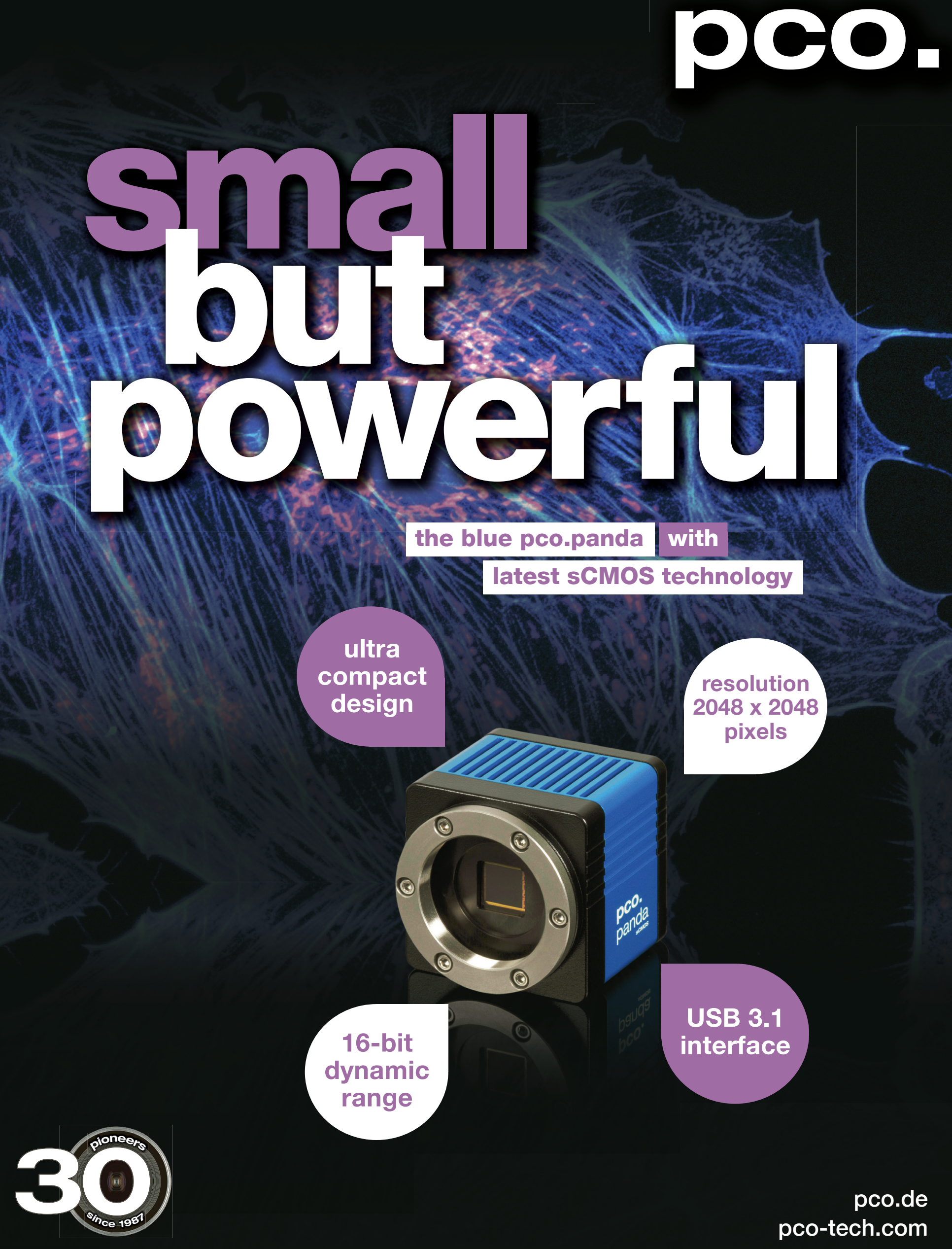


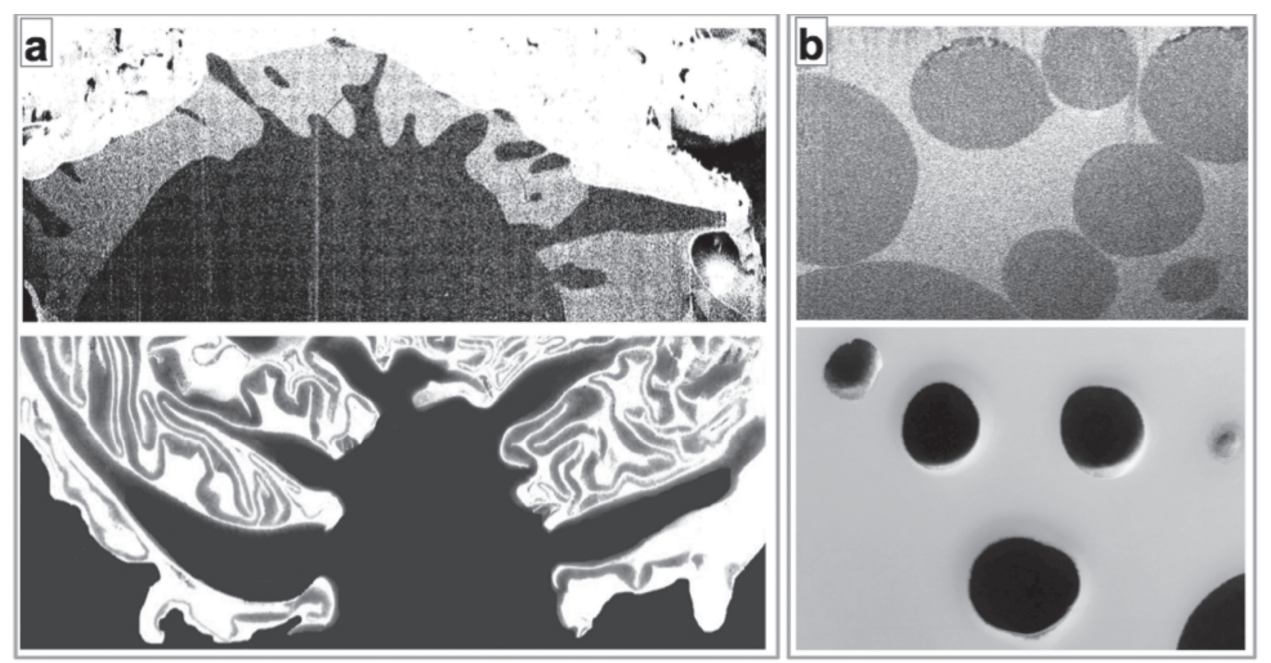

Figure 2: Comparison between grayscale cross sections of (a) nano-icicles covered with oil on anti-frosting surface [19] and red cabbage; and (b) emulsion of microscale water droplets and oil [20] and a slice of Swiss cheese with large holes.

of the object and capturing an image of the exposed cross section. By "slicing-and-viewing" through the entire object of interest, a set of images of sequential cross sections of the object may be collected. These data can be processed using a variety of image processing programs to yield a $3 \mathrm{D}$ reconstruction of the original object that can be used to explore the internal geometry and quantify important characteristics such as secondary phase distribution within a parent phase (for example, nanoparticles in a composite or drops in an emulsion) or porosity (for example, within shells, rocks, or novel 3D electrodes for batteries).

In a manner analogous to these research procedures, we show that 2D images of sequential cross sections of several foods can be quickly reconstructed into a volumetric data set, shared, and interactively explored online using simple and fast image processing. We describe the entire process and present the results of destructive tomography of red cabbage and Swiss cheese. We selected these two common food items because their cross-sectional morphology resembles, albeit at a much larger size, objects that we have previously encountered in our research. For example, the grayscale cross sections of the red cabbage resemble those of nano-icicles covered by oil on anti-frosting coatings [19] (see Figure 2a). Similarly, a grayscale image of holes in Swiss cheese resembles that of water microdroplets and oil emulsion [20] (see Figure 2b). The rich morphology of the red cabbage also resembles geometry of biological cells interacting with nanomaterials imaged using FIB-SEM [21]. Consequently, the proposed activity can be not only used to introduce students to tomography, but can also be a hands-on component of a broader outreach program covering a variety of topics. In the last part of this article, we describe our experience with implementation of this activity in a local middle school.

\section{Materials and Methods}

Red cabbage. We selected a red cabbage about $10 \mathrm{~cm}$ in diameter as our first sample because of its rich internal composition and high contrast between the lighter and darker colors. In order to repeatedly cut thin slices with uniform thickness we employed a mandolin slicer shown with cabbage slices in Figure 3a. After each cut we placed the cabbage in a marked position in front of a white background set by a sheet of paper and captured a digital image of the cross section.

The collected images were pre-processed using ImageJ, Microsoft Windows Paint, or Adobe Photoshop. Specifically, besides adjusting contrast and brightness, the out-of-focus areas outside of the cross section were removed (otherwise the cabbage attains a half-cylinder shape after reconstruction). The images in Figure $3 \mathrm{~b}$ show examples of four "cleaned-up" cabbage sections, in original color and after binarization followed by color inversion (watch Video 1 online in the digital edition of this issue at this web address: https://doi.org/10.1017/S1551929517000839). At this stage of processing of a typical FIB-SEM data set, the grayscale images would be imported into 3D analysis software. The sequential slices would be aligned with respect to each other, segmented into individual phases (for example, using thresholding to produce binary images such as those in Figure 3b), and reconstructed in three dimensions. While conceptually straightforward, full execution of this image processing methodology can be cumbersome and is beyond scope of a $\mathrm{K}-12$ outreach activity. After evaluating several alternatives, we found that ImageJ as well as Wolfram Mathematica can be used relatively easily to conduct a simplified image processing procedure. Since ImageJ is commonly used by microscopists, the image processing steps in this software will not be reiterated here. In turn, Mathematica is commonly available to university students and is being used increasingly in $\mathrm{K}-12$ grades, but it is not commonly used by microscopists. We found that just a few lines of code in this software are needed to make an interactive and easy-to-explore $3 \mathrm{D}$ reconstruction of the original object from the collected images. The sample code for processing the images can be downloaded from Reference [22]. Examples of two progressive cuts in the $x-y$ plane into a red cabbage reconstructed using this code along the $z$-axis are shown in Figure 3c. Along with the 3D reconstruction, these few lines of code produce sliders that a viewer can drag to progressively "clip-away" sections of the object along the $z$-axis as well as the $x$-axis (see Figure $3 \mathrm{~d}$ and watch Video 2 online in the digital edition of this issue at this web address: https://doi.org/10.1017/S1551929517000839). The results can be saved as a Mathematica Demonstration or in Computational Document Format (CDF) and shared via any website (for example, see Reference [22]).

Pre-sliced cheese. Next, in order to improve the safety of the procedure, we looked for samples that can be "pre-sliced" 

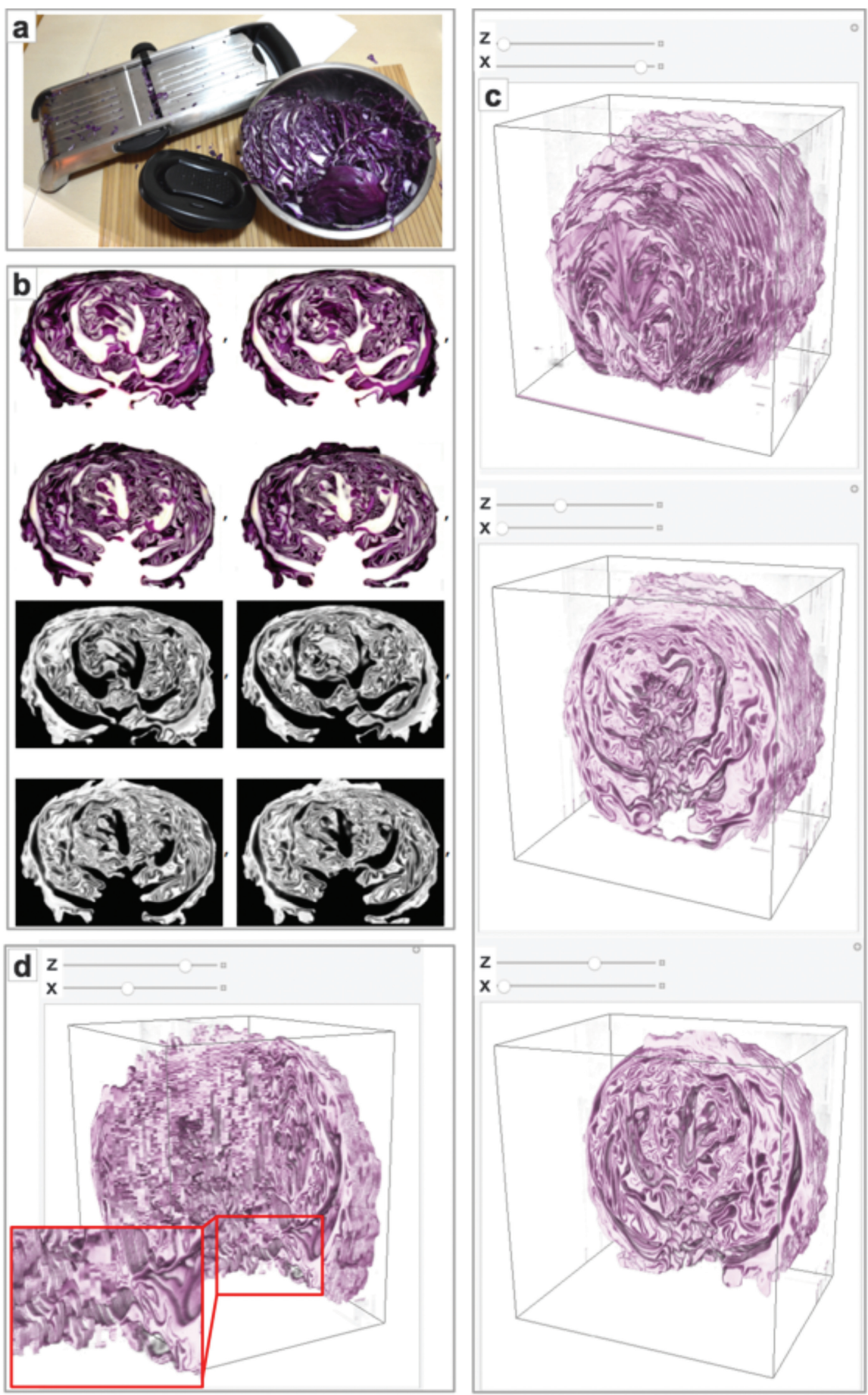

Figure 3: (a) Macroscale "destructive tomography" setup: red cabbage and mandolin slicer; (b) example color and binarized cross sections of the red cabbage; (c) and (d) 3D reconstruction of the red cabbage. (c) shows "cut-aways" along the z-axis, and (d) shows a z-axis and x-axis square cut-away. eliminates the need to spend time "cleaning" the images. In addition, cutting along a side of the rectangular cheese block ensures that each cross section/slice has the same area, removing the need to manually delete out-of-focus portions of the image. Images in Figure 4c show a 3D reconstruction of the cheese block achieved using the same code as the cabbage reconstruction. In addition to reconstructing the full geometry, simple binarization and color inversion can be used to extract the shape of the holes (Figure 4d). Based on the reconstruction of the 3D distribution of the holes, the Swiss cheese that was used had a hole density of $\sim 0.0023$ holes per $\mathrm{cm}^{3}\left(\sim 18\right.$ holes per $\left.800 \mathrm{~cm}^{3}\right)$.

\section{Results: Destructive Tomography Activity in Middle School}

Demonstration program. In the Spring of 2017 undergraduate student interns from ASU's Science Is Fun educational outreach group, which is a part of the LeRoy Eyring Center for Solid State Science [7], implemented the destructive tomography instructional activity at Madison Park Middle School in Phoenix, Arizona. In this activity, three classroom rotations of about thirty 7th grade students each were introduced to the basic concepts of wavelength, imaging resolution, electron microscopy, focused ion beam, and 3D image reconstruction through an engaging 45-minute demonstration that included destructive tomography of red potatoes. The primary goals of this activity were to introduce students to the tools scientists use in a microscopy lab and to provide examples of how these tools are used. The theoretical framework of this instructional activity was based on the educational philosophy of constructivism, in which learning is derived from experience and assessment [23]. Demonstrations in the activity were therefore designed to emphasize student engagement (touching, experimenting) and exploration through questioning rather than repetition of presented facts.

Image resolution. The presentation was structured as a brief introduction to and reassembled prior to giving the "sample" to students. We found that a large block of Swiss cheese is ideal for this purpose. The images in Figures $4 \mathrm{a}$ and $4 \mathrm{~b}$ show an individual slice of the cheese and the $8 \mathrm{~cm} \times 10 \mathrm{~cm} \times 10 \mathrm{~cm}$ block of the cheese after slicing and "reassembling," respectively. Putting each slice of cheese in the same corner of the sheet of paper placed under a camera on a tripod ensures that all images are well aligned. Furthermore, the white background within the holes background concepts ( $5 \mathrm{~min}$ ), followed by a series of discussion questions with multiple short demonstrations (15 min), leading to the primary activity (15 minutes), and a concluding discussion with questions (10 minutes). After the brief discussion and few short activities on the electromagnetic spectrum and image resolution, students were asked, "What do we use to see things that are smaller than the wavelength of our visible light source?" We observed that, using a diagram of the electromagnetic 

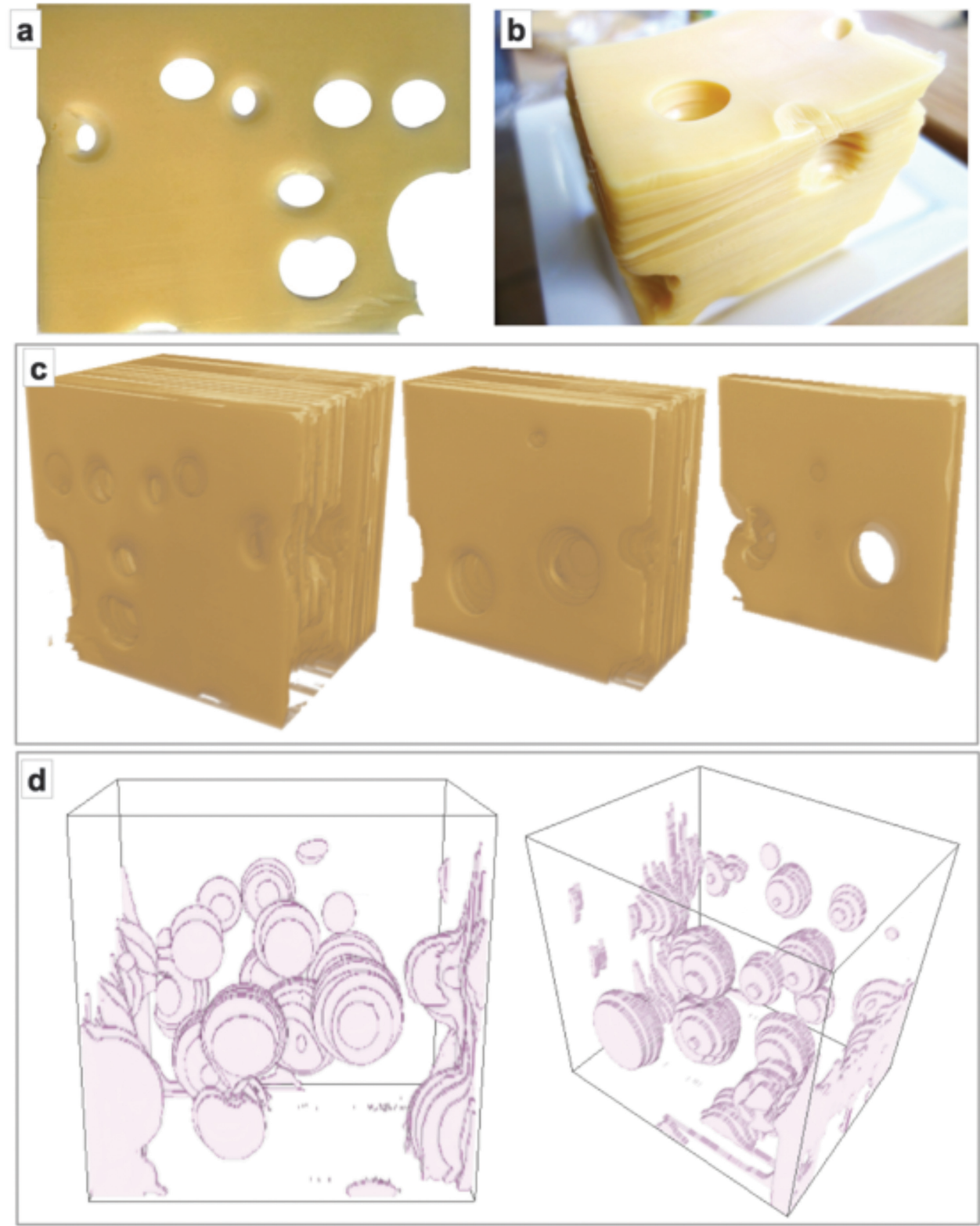

Figure 4: Images of Swiss cheese: (a) an individual slice on a sheet of paper, (b) entire cheese block after slicing and reassembly, (c) 3D reconstruction of the Swiss cheese with three cut-aways along the z-axis, and (d) representation of the hole distribution within the Swiss cheese.

spectrum, many students were able to guess "electrons," which naturally transitioned the discussion onto how an SEM works. The students were then asked to hypothesize what the SEM might be used to image, and they were shown a short video about examining an insect wing sample in the SEM.

Seeing inside. To introduce the FIB, the class was asked how they could see the inside of an object, such as a brain, if the SEM produced only surface images. Exhibiting more confidence in their hypotheses and increased enthusiasm, many of the students responded "Cut it open!" Facilitators then showed students SEM images from a recent study of a crosssectioned synaptic connection of a rat brain [24], and discussed with them how individual slices can be obtained using a microtome or a FIB. The latter discussion was accompanied by display of an animation that explains the typical FIB-SEM procedure [25]. Then a critical question was posed to the students: "What can we learn from these cross-sectional images?" Students had many excellent responses including learning about how the brain works and studying diseases (for example, Alzheimer's disease). Additional uses of the FIB were also discussed in terms of how circuits are produced on computer chips, at which time students were encouraged to use the hand-held magnifying glasses on their desks to observe examples of circuits from older Intel microprocessors.

Tomography. In the final activity, the students sliced and imaged a red potato and were shown how multiple 2D images can be compiled into a volumetric data set. The students first created alignment markers by skewering two central points in the red potato. Then they used the mandolin slicer with a safety guard and Kevlar cut-resistant gloves to cut $2.5 \mathrm{~mm}$ thick slices. The students aligned the slices against an illuminated background and used a phone camera placed on a fixed stand to take the consecutive slice images. The images were then downloaded through a Google Drive App on the smartphone and sent to the facilitator's laptop preloaded with ImageJ, which was used for image processing.

\section{Discussion}

While themed around microscopy, this activity introduced the students to a variety of interdisciplinary concepts and thus could be adapted to work with activities centered around other topics. With some additional software development, an opportunity might arise for combining the described 3D imaging procedure with 3D printers, increasingly popular in $\mathrm{K}-12$ settings. An example might include introduction of students to 3D tomography along with "scaling up" via 3D printing of a dataset of interest obtained with a FIB-SEM (for example, internal components of a cell for biology classes).

\section{Conclusion}

We introduced an experimental methodology for performing macroscale "destructive tomography" of common foods, which can be used as a hands-on activity for K-12 students. The sets of collected 2D images can be processed into volumetric data sets using, for example, ImageJ or Mathematica. We demonstrated our procedure on several foods including a red cabbage, Swiss cheese, and, in a brief in-class activity, a red potato. We also developed an example educational activity that incorporated this destructive 
tomography procedure. This activity was tried by three classroom rotations of about thirty 7 th grade students each in a local middle school. During the activity, student attitudes reflected high levels of engagement (for example, asking questions, expressing excitement, handling of tools and materials), which was confirmed by feedback from observing instructors.

\section{References}

[1] High School SEM Group, "HS_SEM (High Schools with Scanning Electron Microscopes," http://www.hssemgroup. com (accessed July 26, 2017).

[2] C Queenan et al., Microscopy Today 19 (2011) 48-52.

[3] C Queenan et al., SPIE Defense, Security, and Sensing, International Society for Optics and Photonics, (2012) 837811-873820.

[4] C Queenan et al., Microsc Microanal 18 (2012) 294-95.

[5] Bergen County Technical Schools, http://bcts.bergen.org/ index.php/research/nano-structural-imaging-lab (accessed July 26, 2017 https://research.bergen.org/nsil-lab-tour).

[6] T Coffey et al., Microscopy Today 23 (2015) 44-7.

[7] Arizona State University, LeRoy Eyring Center For Solid State Science, "Science is Fun," http://le-csss.asu.edu/ scienceisfun (accessed July 26, 2017).

[8] S Robinson et al., Microscopy Today 19 (2011) 46-50.

[9] A Chumbley and L Chumbley, Microscopy Today 18 (2010) 42-5.

[10] MG Jones et al., Journal of Research in Science Teaching 40 (2003) 303-22.

[11] C Schooley, Microscopy Today 20 (2012) 42-4.

[12] E Elert, Microscopy Today 19 (2011) 38-41.

[13] F Stevie et al., Introduction to focused ion beams: instrumentation, theory, techniques and practice, Springer, New York, 2005.

[14] N Bassim et al., MRS Bulletin 39 (2014) 317-25.

[15] M Cantoni and L Holzer, MRS Bulletin 39 (2014) 354-60.

[16] PG Kotula et al., MRS Bulletin 39 (2014) 361-65.

[17] K Rykaczewski et al., ACS Nano 6 (2012) 9326-34.

[18] N Antoniou et al., MRS Bulletin 39 (2014) 347-52.

[19] K Rykaczewski et al., Langmuir 29 (2013) 5230-38.

[20] S Anand et al., Soft Matter 11 (2015) 69-80.

[21] R. Wierzbicki et al., PloS one 8 (2013) e53307.

[22] Wolfram Demonstrations Project, "Destructive Tomography of Red Cabbage," http://demonstrations. wolfram.com/DestructiveTomographyOfRedCabbage (accessed July 26, 2017).

[23] Educational Broadcasting Corporation, WNET Education, Concept to Classroom, "Workshop: Constructivism as a Paradigm for Teaching and Learning," http://www.thirteen. org/edonline/concept2class/constructivism/index_sub5. html (accessed July 26, 2017).

[24] G Knott et al., Journal of Visualized Experiments 53 (2011) 2588.

[25] E He et al., Lister Hill National Center for Biomedical Communications, NIH/NLM, "Focused Ion Beam Scanning Electron Microscopy," https://vimeo.com/139031380 (accessed July 26, 2017).

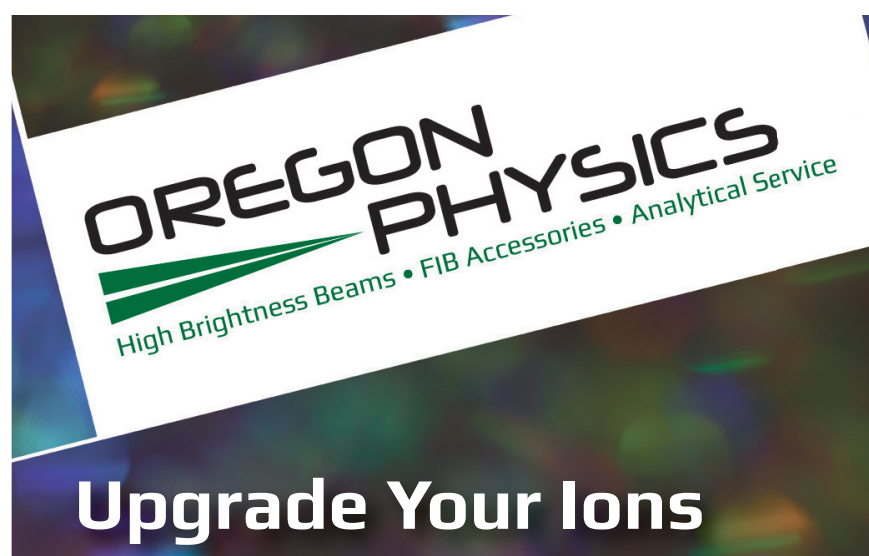

Hyperion $^{\text {TM }}$ Dual Polarity Ion Sources are now available as direct upgrades from Oregon Physics for FEI FIB 200, PHI Adept 1010, and Cameca NanoSIMS, IMS F series, and 12XX series instruments.

\section{Upgrade your ion source to benefit from:}

- Longer source lifetime

- Better image resolution

- Improved depth profiling (SIMS)

- Higher currents for milling (FIB)

Oregon Physics' Hyperion ion sources are designed to bolt-on to your existing optical system for easy implementation.

How will Hyperion improve your research? Learn more at Oregon-Physics.com or call us to discuss your requirements.

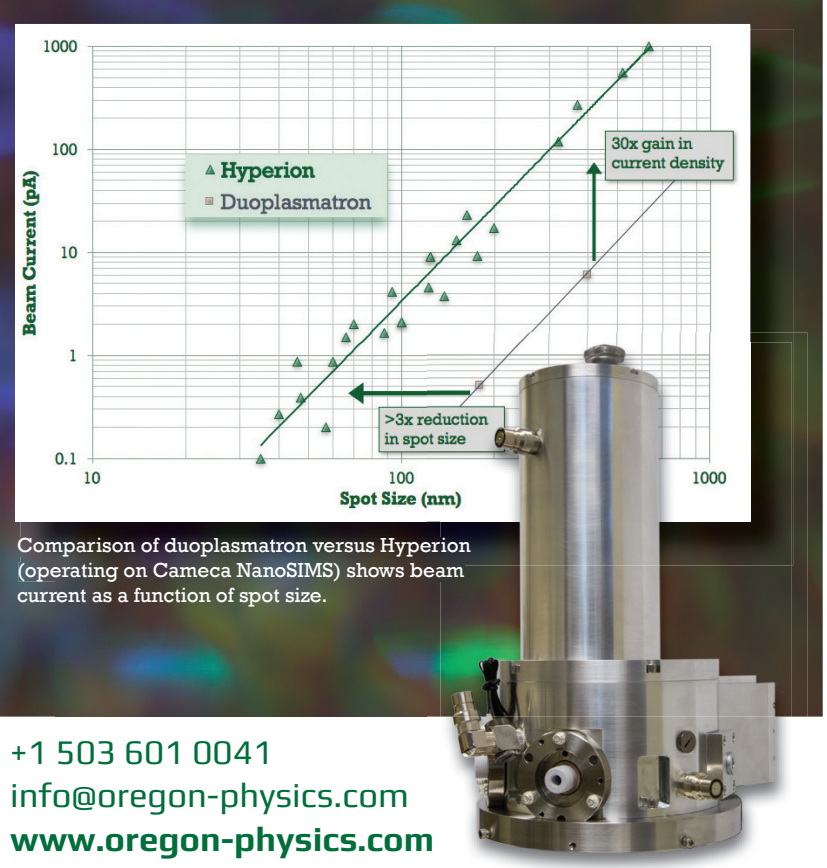

